\title{
ARTICLE OPEN Topological superconductivity in metal/quantum-spin-ice heterostructures
}

\author{
Jian-Huang She ${ }^{1}$, Choong H. Kim $\mathbb{D}^{2}$, Craig J. Fennie ${ }^{2}$, Michael J. Lawler ${ }^{1,3}$ and Eun-Ah Kim ${ }^{1}$
}

We propose a strategy to achieve an unconventional superconductor in a heterostructure: use a quantum paramagnet (QPM) as a substrate for heterostructure growth of metallic films to design exotic superconductors. The proposed setup allows us to "customize" electron-electron interaction imprinted on the metallic layer. The QPM material of our choice is quantum spin ice. Assuming the metallic layer forms a single isotropic Fermi pocket, we predict its coupling to spin fluctuations in quantum spin ice will drive topological odd-parity pairing. We further present guiding principles for materializing the suitable heterostructure using $\mathrm{ab}$ initio calculations and describe the band structure we predict for the case of $\mathrm{Y}_{2} \mathrm{Sn}_{2-x} \mathrm{Sb}_{x} \mathrm{O}_{7}$ grown on the (111) surface of $\mathrm{Pr}_{2} \mathrm{Zr}_{2} \mathrm{O}_{7}$. Using this microscopic information, we predict topological odd-parity superconductivity at a few Kelvin in this heterostructure, which is comparable to the $T_{c}$ of the only other confirmed odd-parity superconductor $\mathrm{Sr}_{2} \mathrm{RuO}_{4}$.

npj Quantum Materials (2017)2:64; doi:10.1038/s41535-017-0063-2

\section{INTRODUCTION}

An intimate connection between a quantum paramagnet (QPM) and superconductivity has long been suspected. Anderson conjectured that a specific type of quantum paramagnet, namely resonating valence bond (RVB) quantum spin liquid could turn into a superconductor upon doping holes in $1987 .^{1}$ The idea uses the notion that a RVB state is a quantum superposition of singlet configurations in which all spins form a singlet with a partner (see Fig. 1a). Such spins simultaneously point in many directions due to quantum fluctuation effects and hence show no sign of magnetic order. Nevertheless widely separated spins in a RVB state maintain a high degree of entanglement driven by the exchange interaction $J_{\text {ex. }}{ }^{2,3}$ Anderson conjectured that a RVB state can turn into a superconducting state by removing spins (doping holes) and allowing singlets to move around, which promote spin singlets to Cooper pairs (see Fig. 1a). However so far there is no material realization of this proposal to the best of our knowledge. Here we propose a conceptually new framework for using a QPM to drive superconductivity without doping or requiring RVB singlets: grow a heterostructure consisting of a QPM and a metal (See Fig. 1b).

We propose to "borrow" the spin correlation of a QPM without destroying the QPM phase. This is conceptually distinct from Anderson's proposal and it has several advantages. Firstly, the superconductor need not be a singlet superconductor. Instead the pairing symmetry now depends on the dynamic spin-spin correlation function and the structure of the interlayer coupling, and hence it can be "chosen" at will through the choice of the QPM layer. Specifically, we will show that the quantum spin ice $e^{3-14}$ as a QPM material will drive topological triplet pairing at the interface. Secondly the two distinct characteristic energy scales of each layers, namely the Fermi energy of the metal $E_{\mathrm{F}}$ (or equivalently $N(0)^{-1}$, the inverse of the density of states at the Fermi level) and the spin-spin exchange interaction of the QPM
$J_{\text {ex }}$, enables us to be in the regime where $J_{\text {ex }} / E_{\mathrm{F}} \ll 1$. Theoretically this small parameter can play the role of $\omega_{\mathrm{D}} / E_{\mathrm{F}} \ll 1$ (where $\omega_{\mathrm{D}}$ represents characteristic phonon frequency) in the Migdal-Eliashberg theory which justifies ignoring a certain set of diagrams and in turn serves as the key to their essentially exact treatment of phonon-mediated superconductivity. ${ }^{15}$ Finally, the coupling between the spins and the itinerant electrons in the form of a Kondo-like coupling $J_{\mathrm{K}}{ }^{16}$ across the interface is expected to be small, i.e., $J_{K} N(0) \ll 1$, making a perturbative treatment in this parameter reliable. These advantages in concert with advances in the atomically precise preparation of relevant heterostructures ${ }^{17-19}$ present an unusual opportunity for a theoretically guided "design" of a new topological superconductor.

Interestingly the problem of coupling between local spinmoments and itinerant electrons has a long and celebrated history itself, especially in the context of heavy-fermion systems. ${ }^{20}$ In the strong coupling limit of $J_{K} N(0) \gg 1$, the conduction electrons hybridize with the local moments to form Kondo singlets resulting in a heavy Fermi liquid ground state (the gray phase labeled HFL in Fig. 1c). On the other hand, in the weak coupling limit $J_{K} N(0) \ll 1$ of our interest, the spins are asymptotically free and there are many more possibilities depending on the strength of the spin-spin exchange interaction $J_{\text {ex }}$. When $J_{\text {ex }}=0$, the RKKY interaction mediated by itinerant electrons, which is a perturbative effect of the coupling to the local moments with the characteristic interaction strength $J_{\text {RKKY }} \sim J_{K}^{2} N(0)$, typically drives an antiferromagnetic order ${ }^{21}$ (see Supplemental Material (SM) Fig. 1). However when $J_{\mathrm{ex}} \neq 0^{22}$ and furthermore frustrated, ${ }^{23}$ such antiferromagnetic ordering will be suppressed. Further, for sufficiently strong $J_{K} N(0)$, the Kondo singlet, the RVB singlet and Cooper pairs may all cooperate to form an exotic superconductor (the purple phase labeled SC $\times$ QPM in Fig. 1c). ${ }^{22-24}$ However, the coupling through the interface would naturally put the proposed heterostructure in

\footnotetext{
${ }^{1}$ Department of Physics, Cornell University, Ithaca, NY 14853, USA; ${ }^{2}$ School of Applied and Engineering Physics, Cornell University, Ithaca, NY 14853 , USA and ${ }^{3}$ Department of physics, Binghamton University, Vestal, NY 13850, USA

Correspondence: Eun-Ah Kim (eun-ah.kim@cornell.edu)
}

Received: 10 July 2017 Revised: 15 September 2017 Accepted: 2 October 2017

Published online: 20 November 2017 


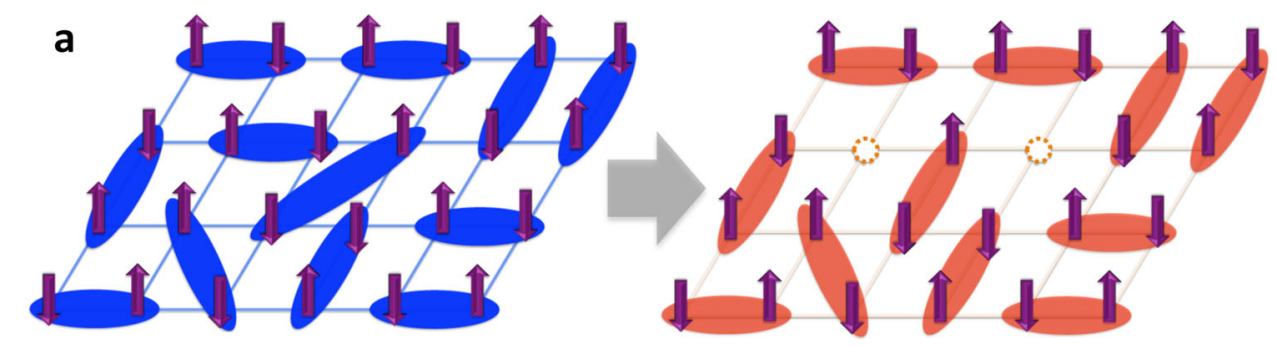

b

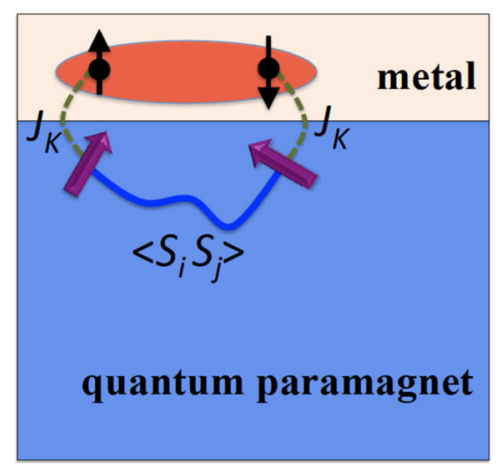

C

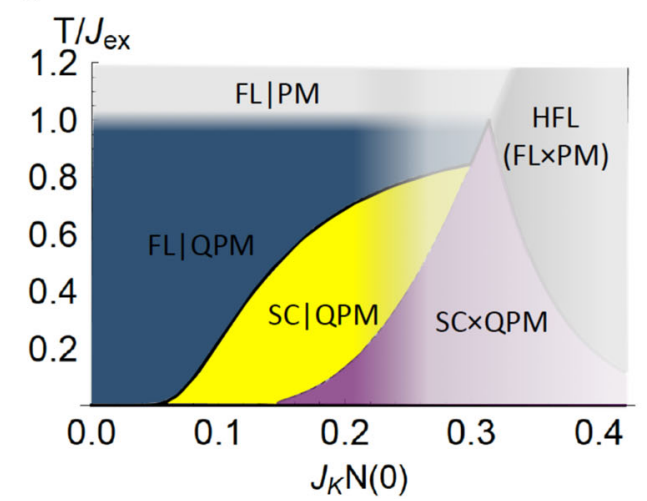

Fig. 1 General considerations of spin-fluctuation-mediated-pairing in the metal/quantum-paramagnet (QPM) heterostructure. a The resonating valence bond (RVB) proposal of unconventional superconductivity by Anderson. ${ }^{1}$ Left represents the parent insulating system where the spins form RVB pairs (blue ellipsoid). By doping holes (dashed circle) into the system, as shown on the right, the RVB pairs become mobile (red ellipsoid), and the whole system becomes superconducting. $\mathbf{b}$ The proposed metal/QPM heterostructure. The metal provides the charge carriers and the QPM provides a pairing interaction via quantum paramagnetic spin-fluctuations $\left\langle\vec{S}_{i} \vec{S}_{j}\right\rangle$. The two systems are coupled via a Kondo type coupling $J_{K}$, which generates Cooper pairing among charge carriers (red ellipsoid). c Phase diagram of the metal-QPM heterostructure. In the FLIPM, FLIQPM and SCIQPM phases, the conduction electrons from the metal and the local moments from the substrate coexist, but are decoupled at the mean field level. The conduction electrons form a Fermi liquid (FL) or a superconductor (SC), while the local moments form an incoherent paramagnet (PM) or a coherent QPM. In the HFL and SC $\times$ QPM phases, the conduction electrons and the local moments hybridize and form Kondo singlets. The aim is to design the heterostructure to be in the SCIQPM phase. This phase diagram applies to the parameter region $J_{\mathrm{RKKY}}<\max \left\{J_{\mathrm{ex}}, T_{\mathrm{K}}\right\}$ for all coupling strength $J_{\mathrm{K}} N(0)$ (see SM section SI)

the small $J_{K} N(0)$ region which has not received much attention todate.

For $J_{K} N(0) \ll 1$, the effect of the interfacial coupling on the metal can be treated perturbatively. Moreover when the QPM has a gapped spectrum with the gap scale $\omega_{\text {sf }} \sim 2 J_{\text {ex }}$, we anticipate the QPM to stay intact on the insulator side (see Fig. 1c) as long as $J_{\text {RKKY }} \sim J_{K}^{2} N(0)<J_{\text {ex }}$. Under these conditions, one can safely "integrate out" the local spin degrees of freedom to arrive at an effective interaction for the itinerant electrons. In the absence of the $J_{\mathrm{K}}$ coupling, the ground state for the heterostructure will consist of decoupled and coexisting Fermi liquid, and QPM for a trivial reason (labeled FL|QPM in Fig. 1c. This phase corresponds to the so-called $\mathrm{FL}^{*}$ phase of refs. 23,25 ). However the Fermi liquid state of the metal may be unstable against ordering once the effective electronic interaction due to the coupling $J_{\mathrm{K}}$ is taken into account. In the absence of Fermi surface nesting, the only such instability that is accessible at infinitesimal coupling strength is a superconducting instability. ${ }^{26}$ Hence as long as $J_{\text {RKKY }}<J_{\text {ex }}$, we anticipate the ground state in the $J_{K} N(0) \ll 1$ regime to consist of superconducting itinerant electrons from the metallic side coexisting with the QPM. This interfacial superconductor, which we dubbed a SC|QPM phase (the yellow phase in Fig. 1c), will be the focus of the rest of this paper.

\section{RESULTS}

In order to materialize the SC|QPM phase, we propose to grow a metallic layer on a QPM substrate to form a coherent interface. The goal will be for each part of the heterostructure to be individually well-understood and to provide one of two essential ingredients of superconductivity: the charge carriers (from metal) and the pairing interaction (from QPM). The growing list of QPM materials and the diversity of their measured spin structure factors make the metal/QPM heterostructure a powerful facility for generating new classes of superconductors (see SM section SII for a list of candidate QPM materials). While our approach applies broadly to all QPM materials, to be concrete we will focus on a class of QPM materials known as the quantum spin ice (QSI) family. The QSI materials are frustrated pyrochlore magnets that not only show no sign of order down to low temperatures, but also exhibit quantum dynamics. ${ }^{3-14}$ Specifically we will focus on $\mathrm{Pr}_{2} \mathrm{Zr}_{2} \mathrm{O}_{7}$ for concreteness and for its appealing properties. Experimentally, $\mathrm{Pr}_{2} \mathrm{Zr}_{2} \mathrm{O}_{7}$ exhibits QPM phenomenology over a large temperature window $(T<1.4 \mathrm{~K})$. For our purpose, the advantage of $\mathrm{Pr}_{2} \mathrm{Zr}_{2} \mathrm{O}_{7}$ over other QPM materials is that the measured dynamical structure factor ${ }^{14} \mathrm{Xab}(\boldsymbol{q}, \omega)$ appears to be well characterized by hydrodynamic theories ${ }^{27-30}$ initially developed for classical spin ice (CSI). CSI obeys the ice rule at zero temperature which amounts to the divergence-free constraint, i.e., $\nabla \cdot \vec{S}(\boldsymbol{r})=0$ for the coarse-grained spin field $\vec{S}(\boldsymbol{r})$. This can be elegantly expressed, ${ }^{27,28}$ in terms of a vector potential $\vec{A}(\boldsymbol{r})$. Quantum or thermal fluctuations introduce an ice rule breaking longitudinal component leading to dynamical susceptibility of the form

$$
X_{\mathrm{ab}}(\mathbf{q}, \omega)=\frac{X_{0}^{\top}}{1-i \omega / \Omega}\left(\delta_{\mathrm{ab}}-\frac{q_{\mathrm{a}} q_{\mathrm{b}}}{q^{2}}\right)+\frac{X_{0}^{\mathrm{L}}}{1-i \omega / \Omega+q^{2} \xi^{2}} \frac{q_{\mathrm{a}} q_{\mathrm{b}}}{q^{2}},
$$


within hydrodynamic theories. ${ }^{27-30}$ Here $\Omega$ is the characteristic energy scale of the dynamics and $\xi$ is the correlation length, $X_{0}^{T / L}$ are overall scales of the transverse (ice-rule preserving) and longitudinal (ice-rule breaking) components respectively, and $X_{0}^{\top}=X_{0}^{\mathrm{L}}$. We find this form provides a good fit to the dynamical susceptibility of $\operatorname{Pr}_{2} \mathrm{Zr}_{2} \mathrm{O}_{7}$ where its quantum nature is manifest in the value of $\Omega$ (see SM section SIII A). Unlike CSI materials with essentially vanishing $\Omega_{1}{ }^{31}$ in $\operatorname{Pr}_{2} \mathrm{Zr}_{2} \mathrm{O}_{7} \Omega \approx 0.17 \mathrm{meV}$ as $T \rightarrow 0 .{ }^{14}$ Since $\Omega$ sets the scale of the superconducting $T_{c}$ (see SM section $\mathrm{SI})$, appreciable dynamics of QSI materials is essential for our proposal.

We first consider the relevant low energy effective theory $H=$ $H_{\mathrm{c}}+H_{\mathrm{s}}+H_{\mathrm{K}} \cdot H_{\mathrm{c}}$ describes the metallic layer with an isotropic Fermi surface:

$$
H_{\mathrm{c}}=\sum_{\boldsymbol{k} a}\left(\frac{\hbar^{2} k^{2}}{2 m}-E_{\mathrm{F}}\right) \psi_{a}^{\dagger}(\boldsymbol{k}) \psi_{a}(\boldsymbol{k}),
$$

where $\psi_{a}^{\dagger}(\boldsymbol{k}) \quad\left(\psi_{a}(\boldsymbol{k})\right)$ creates (annihilates) an electron with momentum $\boldsymbol{k}$ and spin index $a, m$ is the electron's mass and $E_{\mathrm{F}}$ is the Fermi Energy. The spin Hamiltonian $H_{s}$ for the QSI substrate in isolation encodes the above hydrodynamic theory. Finally the dynamic degrees of freedom of each side will couple at the interface through the coupling term $H_{\mathrm{K}}$ (see SM section SIV for discussion of other possible coupling terms). Specifically, we consider a Kondo-like coupling ${ }^{16}$ between the conduction electron spin density $\vec{s}(\boldsymbol{r}, t)=\sum_{\alpha \beta} \psi_{a}^{\dagger}(\boldsymbol{r}, t) \vec{\sigma}_{\alpha \beta} \psi_{\beta}(\boldsymbol{r}, t)$ and the coarse-grained spin operator $\vec{S}(\boldsymbol{r}, t),{ }^{27,28}$ Given the form of dynamical susceptibilitiy of Eq. (1), it turns out that pairing channel remains the same for any finite $\xi$ with manifestly attractive interaction in the $p$-wave channel (see SM section SIV). In order to elucidate this unusual mechanism we focus on the transverse components of the spin-field, for which the simplest form of interfacial coupling reads

$$
\begin{aligned}
H_{\mathrm{K}} & =J_{\mathrm{K}} v_{\text {cell }} \sum_{a a \beta} \int d^{2} \boldsymbol{r} \psi_{a}^{\dagger}(\boldsymbol{r}) \vec{\sigma}_{a \beta} \psi_{\beta}(\boldsymbol{r}) \cdot \vec{S}\left(\boldsymbol{r}_{\perp}=\boldsymbol{r}, \boldsymbol{z}=0\right) \\
& =-J_{\mathrm{K}} v_{\mathrm{cell}} \sum_{a a \beta} \int d^{2} \boldsymbol{r}\left(\vec{\nabla} \times \psi_{a}^{\dagger}(\boldsymbol{r}) \vec{\sigma}_{a \beta} \psi_{\beta}(\boldsymbol{r})\right) \cdot \vec{A}\left(\boldsymbol{r}_{\perp}=\boldsymbol{r}, z=0\right)+\cdots,
\end{aligned}
$$

upon integrating by parts. Here $\vec{\sigma}$ denotes the Pauli matrices, $v_{\text {cell }}$ the volume of the unit cell, $z=0$ the interface, and $\cdots$ represents coupling with longitudinal components of the spin field. Notice the rather obvious form of the coupling in the spin language takes a rather unusual form in the vector potential language. Usually when fermions are "charged" under a vector potential associated with a gauge symmetry, it couples minimally via $\vec{j} \cdot \overrightarrow{\mathcal{A}}$ coupling where the fermion current is $\vec{j} \equiv Q \frac{\boldsymbol{k}}{m} \psi_{\boldsymbol{k} a}^{\dagger} \psi_{\boldsymbol{k} a}$ with $Q$ being the charge of the fermion field $\psi$ with respect to the gauge field $\overrightarrow{\mathcal{A}}$. Interestingly it is well established that gauge field dynamics hinder pairing under such circumstances. ${ }^{32,33}$ The unusual form of coupling between the electrons and vector potential in Eq. (3) in the form of $(\vec{\nabla} \times \vec{S}) \cdot \vec{A}$ is due to the fact that the magnetic monopoles, rather than electrons are elementary excitations that would couple minimally to the vector potential $\vec{A}$. This exotic coupling has striking consequences when we consider pairing possibilities.

In the regime of interest, the leading effect of the coupling (3) on the spin physics is to induce the RKKY interaction $J_{\text {RKKY. }}$. However, as long as the electrons superconduct at a temperature above $J_{\mathrm{RKKY}} / k_{\mathrm{B}}$, subtleties induced by this interactions can be neglected. Then we can "integrate out" the dynamical spin fluctuaitons and focus on the effect of the interaction induced on the metallic layer. Given a QPM substrate $(\langle\vec{S}(r, t)\rangle=0$ by definition), the leading effect of the coupling Eq. (3) is to induce a four-fermion interaction term in the effective action (see SM section $\mathrm{SI}-\mathrm{B}$ ) in the path integral representation. Resorting to the Hamiltonian notation, one has:

$$
H_{\text {int }}(t)=-\frac{J_{K}^{2} v_{\text {cell }}^{2}}{2 \hbar} \int d t^{\prime} \int d^{2} \boldsymbol{r} d^{2} \boldsymbol{r}^{\prime} \vec{s}(\boldsymbol{r}, t) \cdot\left\langle\vec{S}(\boldsymbol{r}, 0, t) \vec{S}\left(\boldsymbol{r}^{\prime}, 0, t^{\prime}\right)\right\rangle \cdot \vec{S}\left(\boldsymbol{r}^{\prime}, t^{\prime}\right)
$$

$$
\begin{gathered}
\simeq-\frac{\int_{K}^{2} v_{c e l l}^{2}}{2 \hbar} \sum_{\mathrm{ab} \alpha \beta \alpha^{\prime} \beta^{\prime}} \int d t^{\prime} \int d^{2} \boldsymbol{r} d^{2} \boldsymbol{r}^{\prime}\left[\left(\vec{\sigma}_{a \beta} \times \vec{\nabla}\right)_{\mathrm{a}} \psi_{\boldsymbol{r} a}^{\dagger} \psi_{\boldsymbol{r} \beta}\right] \\
\mathcal{D}_{\mathrm{ab}}\left[\left(\vec{\sigma}_{\alpha^{\prime} \beta^{\prime}} \times \vec{\nabla}\right)_{\mathrm{b}} \psi_{\boldsymbol{r}^{\prime} \alpha^{\prime}}^{\dagger} \psi_{\boldsymbol{r}^{\prime} \beta^{\prime}}\right]
\end{gathered}
$$

where $\mathcal{D}_{\mathrm{ab}}\left(\boldsymbol{r}-\boldsymbol{r}^{\prime}, t-t^{\prime}\right) \equiv\left\langle A_{\mathrm{a}}(\boldsymbol{r}, 0, t) A_{\mathrm{b}}\left(\boldsymbol{r}^{\prime}, 0, t^{\prime}\right)\right\rangle$ represents the vector field propagator, whose classical limit in momentum space $\left\langle A_{\mathrm{a}}(\boldsymbol{q}) A_{\mathrm{b}}(-\boldsymbol{q})\right\rangle \sim \frac{1}{q^{2}}\left(\delta_{\mathrm{ab}}-2 \hat{q}_{\mathrm{a}} \hat{q}_{\mathrm{b}}\right)^{27,28}$ encapsulates the ice-rule. Note that now the quantum correlation between spins of the QPM gets imprinted on the effective interaction between itinerant electrons. Therefore one can "manipulate" the interaction between itinerant electrons through the choice of the QPM. Further Eq. (5) reveals how the exotic form of fermion-vector field coupling (Eq. (3)) specific to QSI-metal heterostructure induces manifestly attractive interaction between electrons of equal spin $\vec{S}(\boldsymbol{r})=\vec{S}\left(\boldsymbol{r}^{\prime}\right)$ that can drive triplet pairing.

Now the low energy effective theory defined by Eqs. (2) and (4) describes an interacting electron problem, which is generically hard to solve. To make the problem worse, the effective interaction Eq. (4) is highly structured as a result of the spin ice rules. However, we can make non-trivial progress building on the renormalization group-based perspectives ${ }^{26}$ and the classic justification for the mean-field theory treatment in the BCS theory. ${ }^{15}$ Firstly, we know from the renormalization group theory that the only weak-coupling instability of a Fermi liquid in the absence of Fermi-surface nesting is the superconducting instability. ${ }^{26}$ Secondly, armed with the separation of scale $\Omega / E_{\mathrm{F}} \ll 1$, we expect the mean-field theory treatment in the pairing channel to yield a reliable prediction for the interacting fermion problem when the interaction is weak, i.e., the eigenvalue of the pairing vertex $\lambda \sim N(0) V \sim J_{K}^{2} N(0) / \Omega<1$. Thirdly, the interaction in Eq. (5) is clearly attractive in the equal-spin pairing channel and hence we can anticipate pairing instability at mean-field level. All together the problem at hand promises an opportunity to predict an exotic superconductor whose pairing channel is determined by spindependent and momentum-dependent interaction of Eq. (5), in a theoretically reliable approach.

Therefore we use mean-field theory to look for pairing. We first note that the symmetry of the effective interaction $H_{\text {int }}$ Eq. (4) can be read off directly from the measured spin susceptibility as shown in Eq. (1). Due to the coupling between the spin direction (a) dependence and the momentum direction (q) dependence in Eq. (1), the total angular momentum and the total spin are not separately conserved in $H_{\text {int }}$. Instead the effective interaction $H_{\text {int }}$ in Eq. (4) reduces the total orbital and spin rotational symmetry of $S O(3) \times S U(2)$ down to $U(1)$ leaving only the $z$-component of the total angular momentum $J_{z}=L_{z}+S_{z}$ a good quantum number for the Cooper pairs. Nevertheless the effective interaction is even under parity leaving parity to be a good quantum number.

Now we seek the dominant pairing channel. For this we will keep the frequency dependence implicit and mean-field decouple $H_{\text {int }}$ using pair operators formed out of $2 \times 2$ matrices in the spin basis $P_{\mu}(\boldsymbol{k}) \equiv \frac{1}{\sqrt{2}} \sum_{a \alpha^{\prime}}\left[i \sigma_{y} \sigma_{\mu}\right]_{a \alpha^{\prime}} \psi_{a}(\boldsymbol{k}) \psi_{a^{\prime}}(-\boldsymbol{k})$, where $\sigma_{\mu}=\llbracket$ for $\mu$ $=0$ and Pauli matrices for $\mu=x, y, z{ }^{34}$ Further since pairing occurs near the Fermi surface, we can focus on the angular $\left(\varphi_{\mathbf{k}}\right)$ dependence of the pair operators and decompose the pair operators into different partial wave components: $P_{\mu}\left(L_{z}\right) \equiv \int \frac{\mathrm{d} \varphi_{\mathbf{k}}}{2 \pi} e^{-i L_{z} \varphi_{\mathbf{k}}} P_{\mu}(\mathbf{k})$. Guided by symmetry we then combine pair operators with different orbital angular momentum $L_{z}$ and total spin $S_{z}$ into the $J_{z}$ basis, and diagonalize the interaction 
a

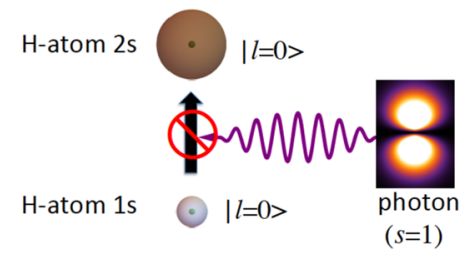

C

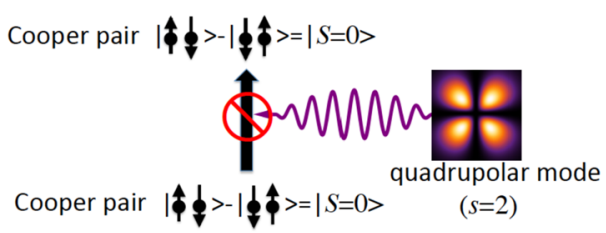

e

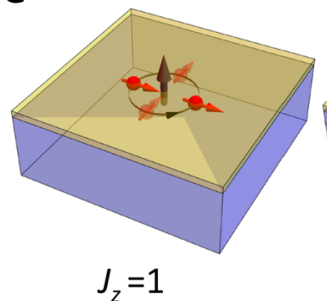

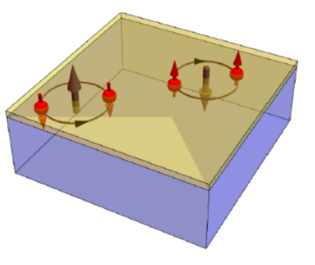

$J_{z}=0$ b

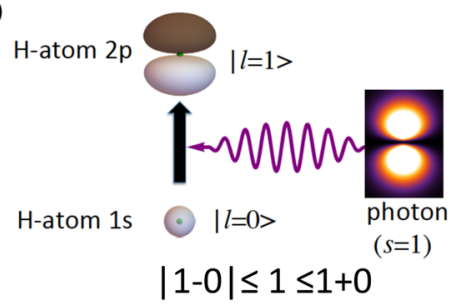

d

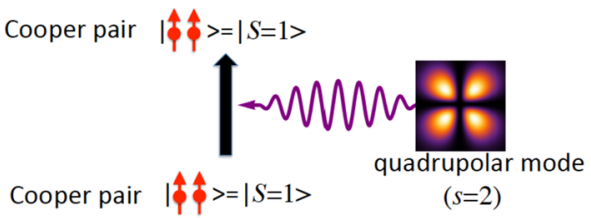

$|2-1| \leq 1 \leq 2+1$

f

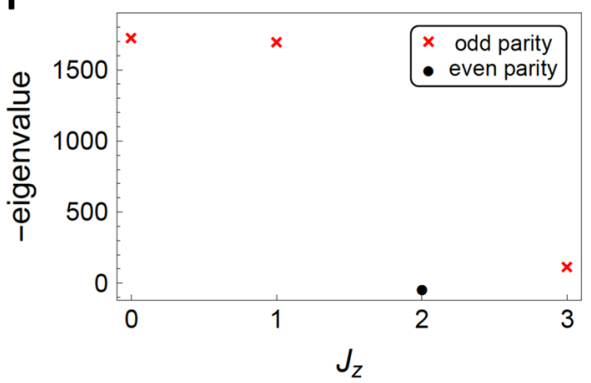

Fig. 2 The dominant pairing channels in the metal/quantum-spin-ice heterostructure. a-d Understanding the emergence of parity-odd spintriplet pairing from selection rules. $\mathbf{a}$, $\mathbf{b}$ represent the dipole transitions for atomic hydrogen: transition from $1 \mathrm{~s}$ state with angular momentum $I=0$ to $2 s$ state also with $I=0$ is forbidden by the selection rule $\left(|1-I| \leq I^{\prime} \leq 1+I\right)$, while transition from $1 s$ state to $2 p$ state with $I=1$ is allowed. c, $\mathbf{d}$ represent the pairing problem under the rank-two magnetic dipole-dipole interaction: spin-singlet pairing with total spin $S=0$ is forbidden by the selection rule $(|2-S| \leq S \leq 2+S)$, while spin-triplet pairing with total spin $S=1$ is allowed. e Illustration of spin and angular momentum configurations of the dominant pairing channels. The larger (brown) arrows represent the orbital angular momenta, and the smaller (red) arrows represent the electron spins. Spin and orbital angular momentum are coupled to yield the total angular momentum $J_{z}=$ 0,1 . $f$ The leading negative eigenvalues of the pairing interaction matrix for different parity and $J_{z}$ channels in the low energy effective model. The eigenvalues are dimensionless numbers in arbitrary units. The dominant pairing channels have odd parity with $J_{z}=0, \pm 1$

Hamiltonian Eq. (5) into

$H_{\text {int }}=\sum_{\kappa= \pm} \sum_{J_{z}=-\infty}^{\infty} V^{(\kappa)}\left(J_{z}\right) P_{J_{z}, \kappa}^{\dagger} P_{J_{z}, \kappa}$,

where $P_{z_{2}, k}$ denotes the pair operator with the $z$-component of total angular momentum $J_{z}$ and parity $k$ (+ for even parity, - for odd parity). (see SM section SI).

Before turning to the numerical results of $V^{(k)}\left(J_{z}\right)$, we can gain much insight by decomposing the pair-wise interaction in Eq. (5) into a rank-2 component, and a rank-0 component:

$H_{\mathrm{int}} \equiv H_{\mathrm{int}}^{(2)}+H_{\mathrm{int}}^{(0)}=\frac{J_{K}^{2}}{8 \pi} \frac{1}{r^{3}}\left[\boldsymbol{s}_{1} \cdot \boldsymbol{s}_{2}-3\left(\boldsymbol{s}_{1} \cdot \hat{r}\right)\left(\boldsymbol{s}_{2} \cdot \hat{r}\right)\right]-\frac{J_{K}^{2}}{3} \delta(\boldsymbol{r}) \boldsymbol{s}_{1} \cdot \boldsymbol{s}_{2}$.

Here $H_{\text {int }}^{(2)} \propto \mathcal{R}^{(2)}\left(\boldsymbol{r}_{1}, \boldsymbol{r}_{2}\right) \cdot S^{(2)}\left(\boldsymbol{s}_{1}, \boldsymbol{s}_{2}\right)$, with rank-two tensors $\mathcal{R}^{(2)}$ 1 and $\mathcal{S}^{(2)}$ (magnetic quadrupole moment) acting on coordinate space and spin space, respectively. The resulting pairing symmetry can be obtained analytically in the strong coupling BEC limit, where the many-body pairing problem reduces to the quantum mechanical problem of diatomic molecules. ${ }^{35}$ First note that $\left\langle H_{\text {int }}^{(2)}\right\rangle=0$ in the even parity channel due to a selection rule (see SM section SIV). The celebrated selection rule that forbids optical transition between $1 s$ and $2 s$ state(Fig. 2a), but allows the transition between $1 s$ and $2 p$ state (Fig. $2 \mathrm{~b}$ ) is an example of how a tensor operator of rank $r, \mathcal{T}^{(r)}$ connects two angular momentum states $|I\rangle$ and $\left|I^{\prime}\right\rangle$ according to the Wigner-Eckart theorem. The theorem states $\left\langle I^{\prime}\left|\mathcal{T}^{(r)}\right| I\right\rangle=0$ unless $|r-l| \leq I^{\prime} \leq(r+I)$. In the case of optical transitions of a hydrogen atom, the tensor operator involved is a rank $r=1$ vector field as the photon is a spin $s=1$ boson. In our case, the scattering potential carries a quadrupolar moment which is a rank $r=2$-tensor. Hence the selection rule forbids pairing in the singlet channel, i.e., $\left\langle S=0\left|H_{\mathrm{int}}^{(2)}\right| S=0\right\rangle=0$ (Fig. 2c). Since the Pauli principle dictates all even parity pairing to be singlet, the selection rule limits possible pairing to the odd-parity channel (Fig. 2d). Second we note that among the odd-parity states,

$$
\left|J_{z}=0\right\rangle \sim\left(k_{x}+i k_{y}\right)|\downarrow \downarrow\rangle+\left(k_{x}-i k_{y}\right)|\uparrow \uparrow\rangle
$$

and

$$
\left|J_{z}= \pm 1\right\rangle \sim\left(k_{x} \pm i k_{y}\right) \frac{|\uparrow \downarrow\rangle+|\downarrow \uparrow\rangle}{\sqrt{2}} \sim k_{y} \frac{|\rightrightarrows\rangle_{x}-|\leftleftarrows\rangle_{x}}{\sqrt{2}} \mp k_{x} \frac{|\rightrightarrows\rangle_{y}-|\leftleftarrows\rangle_{y}}{\sqrt{2}} .
$$

states indeed yield pair-binding (see Fig. 2e and SM section SIV). This is consistent with the earlier insight that Eq. (5) is attractive in the equal-spin pairing channel. On the other hand, the isotropic rank-0 component $H_{\text {int }}^{(0)}$ which can only contribute to $s$-wave channel is purely repulsive, i.e., $\left\langle S=0\left|H_{\mathrm{int}}^{(0)}\right| S=0\right\rangle>0$. Indeed full numerical calculation of interaction strengths $V^{(\mathrm{k})}\left(J_{z}\right)$ confirms the above analysis and predicts the two odd-parity pairing channels 
a

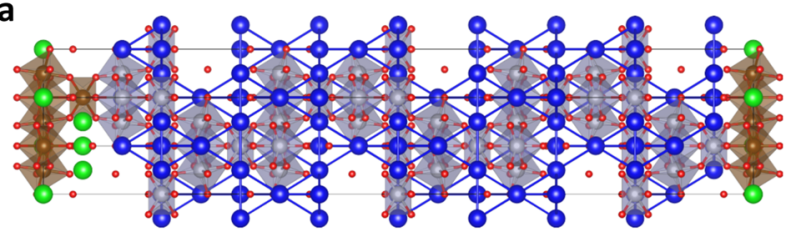

b

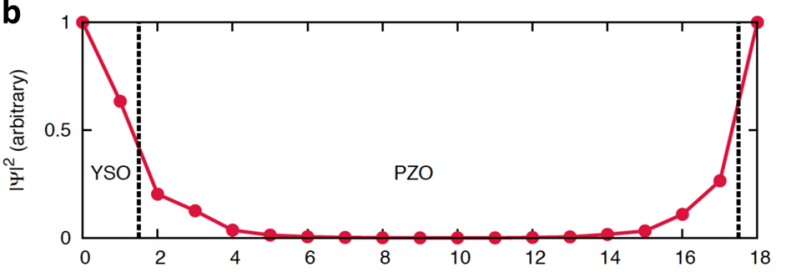

C

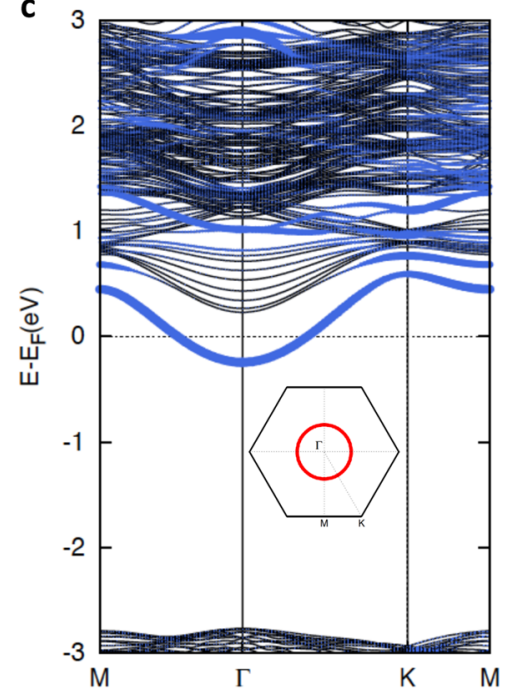

Fig. 3 A concrete material realization of the metal/quantum-spin-ice heterostructure: $\mathrm{Pr}_{2} \mathrm{Zr}_{2} \mathrm{O}_{7} / \mathrm{Y}_{2} \mathrm{Sn}_{2-x} \mathrm{Sb}_{x} \mathrm{O}_{7}$ (111). a Lattice structure. Two layers of Sb-doped $\mathrm{Y}_{2} \mathrm{Sn}_{2} \mathrm{O}_{7}$ deposited on top of 16 layers of $\mathrm{Pr}_{2} \mathrm{Zr}_{2} \mathrm{O}_{7}$ along the [111] direction. The magnetic moments are on the $\mathrm{Pr}$ sites (blue), which form alternating layers of triangular and Kagome lattices. The conduction electrons are donated by the $\mathrm{Sn}$ atoms (brown). Red is O, Green is $\mathrm{Y}$ and Gray is Zr. $\mathbf{b}$ Amplitude of the conduction electron wavefunction in the direction perpendicular to the interface showing penetration into the first two or three layers of $\mathrm{Pr}_{2} \mathrm{Zr}_{2} \mathrm{O}_{7}$. c Band structure, with Fermi surface shown in the inset. There is a single band crossing the Fermi energy, and a single circular Fermi surface around the $\Gamma$ point, with Fermi energy $E_{F} \simeq 0.3 \mathrm{eV}$, and Fermi momentum $k_{F} \simeq 0.37(2 \pi / a)$, where $a$ is the lattice constant of $\mathrm{Pr}_{2} \mathrm{Zr}_{2} \mathrm{O}_{7}$

with $J_{z}=0$ and $J_{z}= \pm 1$ to be overwhelmingly dominant, with predicted $T_{\mathrm{c}} \sim \Omega e^{-1 / \lambda}$, where $\lambda$ is the eigenvalue of the pairing vertex: $\lambda \sim N(0) V^{(\kappa)}\left(J_{z}\right) \sim J_{\mathrm{K}}^{2} /\left(E_{\mathrm{F}} \Omega\right.$ ) (see SM section SI, SIV). Pairing in either of these channels on a single Fermi surface will be topological. ${ }^{36,37}$

There remains the important question of the effect of short distance physics. As we show in SM section SIV through a BCS calculation, the short-distance physics does not alter the pairing symmetry, and its effect on the leading $p$-wave eigenvalues is also mild. This is indeed expected from the fact that the rank- 2 tensor interaction that drives pairing is long-ranged, and hence is not much affected by short distance physics. Short distance physics does substantially renormalize the short-ranged and repulsive rank-0 interaction, reducing it by several orders of magnitude. Thus, the simple model of Eqs. (3) and (5) serves as a faithful representation of the core physics of the real materials.
We now turn to a material proposal for the metallic layer that is expected to fit the above long-wave length description. The requirements of chemical stability at the interface with negligible charge transfer between the two materials and matching of lattice constants restrict the choice of materials. In particular, we need the metallic layer to stably grow with the interface along the (111) surface in order to avoid the generation of orphan bonds and preserve the spin correlations of QSI. Electronically we require the metallic layer to be a good metal without strong Fermi surface nesting, the simplest example being an s-band metal. Such a metal would have several merits: (1) large band width, (2) weak correlation, which helps to avoid ordering by itself; (3) nondegenerate bands, which helps to provide odd numbers of Fermi surfaces to generate topological superconductivity. Unfortunately, existing metallic pyrochlores such as $\mathrm{Bi}_{2} \mathrm{Ru}_{2} \mathrm{O}_{7}$ or $\mathrm{Bi}_{2} \mathrm{Ru}_{2} \mathrm{O}_{7}$ do not satisfy these criteria since they show complicated Fermi surfaces due to the conduction electron of $d$-electron character.

One robust strategy to realize the targeted metallic system is to dope a band insulator with an empty conduction band of $s$ character. Simple crystal chemical rules point to compounds with $\mathrm{Sn}^{4+}$ or $\mathrm{Bi}^{5+}$ at the B-site of $\mathrm{A}_{2} \mathrm{~B}_{2} \mathrm{O}_{7}$, e.g., $\mathrm{Y}_{2} \mathrm{Sn}_{2} \mathrm{O}_{7}$ or $\mathrm{La}_{2} \mathrm{Sn}_{2} \mathrm{O}_{7}$, as prime candidates for the insulating starting material. In practice, doping of the empty conduction band can be performed in a few different ways. For example, one could dope the B-site with $\mathrm{Sb}$ or substitute the A-site with $\mathrm{Ce}^{4+}$ to form, e.g., $(\mathrm{La} / \mathrm{Ce})_{2} \mathrm{Sn}_{2} \mathrm{O}_{7}$. For illustration of the principle, we performed $a b$ initio calculations using density functional theory (DFT) on $\mathrm{Y}_{2} \mathrm{Sn}_{2-x} \mathrm{Sb}_{x} \mathrm{O}_{7} / \mathrm{Pr}_{2} \mathrm{Zr}_{2} \mathrm{O}_{7}$ heterostructures (see Fig. 3a and SM section SIII). In order to minimize the proximity effect which lowers the superconducting gap, we considered one unit-cell thick metallic layer and imposed periodic boundary condition in the direction perpendicular to the interface. As shown in Fig. $3 c$ we find a single circular Fermi surface centered at the $\Gamma$ point for the heterostructure with $\mathrm{Y}_{2} \mathrm{Sn}_{2-x} \mathrm{Sb}_{x} \mathrm{O}_{7}$ at doping level $x=0.2$. Moreover, the conduction electron wavefunction penetrates into the first two layers of $\mathrm{Pr}_{2} \mathrm{Zr}_{2} \mathrm{O}_{7}$ (Fig. 3b), generating a finite Kondo coupling across the interface. Furthermore we have not observed any significant charge transfer from the charge analysis of first-principles calculations. Hence we have ruled out the possibility of the quantum spin ice phase getting destroyed by charge transfer from the metallic layer. Our estimates for the parameters are $E_{\mathrm{F}}=300 \mathrm{meV}, \Omega=0.17 \mathrm{meV}$, $5 \mathrm{meV} \leqslant J_{\mathrm{K}} \leqslant 14 \mathrm{meV}$. Of these, $E_{\mathrm{F}}$ was obtained directly from DFT, $\Omega$ from experiment and $J_{\mathrm{K}}$ from second order perturbation on a realistic atomic Hamiltonian (see SM section SIII). These parameters display a separation of energy scales, i.e. $\Omega / E_{\mathrm{F}} \ll 1$, that ensures the reliability of the above BCS-like treatment of the superconducting instability. Carrying out a microscopic treatment of interfacial superconductivity for the heterostructure of Fig. 3a using the electronic structure of Fig. 3c (see SM section SIV), we confirmed the predictions of the low energy effective model Eqs. (1) and (6) and obtained an estimate of $T_{\mathrm{c}}$ from $T_{\mathrm{C}} \sim \Omega e^{-E_{\mathrm{F}} \Omega / J_{\mathrm{K}}^{2}}$. Using our parameters, this is of order $1 \mathrm{~K}$. The precise value of $T_{c}$ depends sensitively on the dimensionless ratio $\lambda$ which in turn depends on the microscopic details and the actual value of $J_{K}$. We note that the predicted $T_{\mathrm{c}}$ is comparable to the only other solid state candidate for topological superconductivity $\mathrm{Sr}_{2} \mathrm{RuO}_{4}$ with $T_{\mathrm{c}} \simeq 1.5 \mathrm{~K}^{38}$

\section{DISCUSSION}

The strategy we developed here for predictively achieving an exotic superconducting state we dubbed SC|QPM has profound theoretical and experimental consequences. At the level of theoretical principle it is a new approach to drive superconductivity from a quantum paramagnet. Our proposal to borrow the quantum spin fluctuation of a quantum paramagnet ${ }^{3}$ to form exotic superconductors through heterostructure growth bears similarities to excitonic fluctuation proposals. ${ }^{39-43}$ However, the 
latter charge fluctuation-based mechanism suffers from various issues. For instance, tunneling of electrons from the metal can damage the small charge gap of the semi-conductor and kill the charge fluctuation. Further, the local charge fluctuations only drives s-wave pairing which needs to overcome the Coulomb repulsion. By utilizing the goodness of the quantum paramagnet, i.e., spin-fluctuation, our proposal bypasses these issues. At the level of a specific choice of quantum spin-ice, we demonstrated how vector-potential can mediate attractive interaction in the triplet channel through unconventional coupling between the vector field and the fermions, which is nevertheless natural coupling in the language of spins. Our concrete material proposal can guide experimental pursuit of the proposed heterostructure. Clearly the experimental control over atomic interfaces and thin films have reached the state that can support superconductivity. ${ }^{17,18,44-46}$ Likely the biggest challenge will be in controlling disorder which is unavoidable with chemical doping for the metallic layer. Alternatively one may employ a field-effecttransistor (FET) type setup, either using conventional electricfield gating ${ }^{47}$ or using ionic liquid gating. ${ }^{48,49}$ In practice, chemical doping and electric-field gating can be combined to achieve optimal efficiency. Successful materialization of the proposed topological superconductor will be a major breakthrough in superconductivity research, as well as in the application of quantum paramagnets including quantum spin liquids.

\section{Data availability}

The data supporting the findings of this study are available from the corresponding authors on reasonable request.

\section{ACKNOWLEDGEMENTS}

The authors acknowledge useful discussions with Leon Balents, Steve Kivelson, SungBin Lee, Tyrel McQueen and Arun Paramekanti. The authors are grateful to Michel Gingras and Darrell Schlom for a careful reading of the manuscript and helpful comments and suggestions. J.-H.S. acknowledges support by the U.S. Department of Energy, Office of Basic Energy Sciences, Division of Materials Science and Engineering under Award DE-SC0010313; E.-A.K. acknowledge support from the W.M. Keck Foundation; C.H.K. and C.J.F. acknowledge support by NSF Grant No. DMR-1056441.

\section{AUTHOR CONTRIBUTIONS}

J.-H.S. carried out long-wave-length effective theory calculations as well as lattice mean-field theory calculations and analyzed the different pairing channels. C.H.K. identified candidate metallic layer materials and carried out the ab-initio calculations of the electronic structure for the heterostructures of interest. C.J.F., M.J.L. and E.-A. K supervised the project and wrote the paper with contributions from C.H.K. and J.-H.S.

\section{ADDITIONAL INFORMATION}

Supplementary information accompanies the paper on the npj Quantum Materials website (https://doi.org/10.1038/s41535-017-0063-2).

Competing interests: The authors declare that they have no competing financial interests.

Publisher's note: Springer Nature remains neutral with regard to jurisdictional claims in published maps and institutional affiliations.

\section{REFERENCES}

1. Anderson, P. W. The resonating valence bond state in la2cuo4 and superconductivity. Science 235, 1196-1198 (1987).

2. Lee, P. A. An end to the drought of quantum spin liquids. Science 321, 1306-1307 (2008).

3. Balents, L. Spin liquids in frustrated magnets. Nature 464, 199-208 (2010).

4. Gingras, M. J. P. \& McClarty, P. A. Quantum spin ice: a search for gapless quantum spin liquids in pyrochlore magnets. Rep. Prog. Phys. 77, 056501 (2014).

5. Savary, L. \& Balents, L. Quantum spin Liquids. Rep. Prog. Phys. 80, 016502 (2017).
6. Hermele, M., Fisher, M. P. A. \& Balents, L. Pyrochlore photons: the u(1) spin liquid in a $\mathrm{s}=$ three-dimensional frustrated magnet. Phys. Rev. B 69, 064404 (2004).

7. Onoda, S. \& Tanaka, Y. Quantum melting of spin ice: emergent cooperative quadrupole and chirality. Phys. Rev. Lett. 105, 047201 (2010).

8. Ross, K. A., Savary, L., Gaulin, B. D. \& Balents, L. Quantum excitations in quantum spin ice. Phys. Rev. X 1, 021002 (2011).

9. Lee, S., Onoda, S. \& Balents, L. Generic quantum spin ice. Phys. Rev. B 86, 104412 (2012).

10. Benton, O., Sikora, O. \& Shannon, N. Seeing the light: experimental signatures of emergent electromagnetism in a quantum spin ice. Phys. Rev. B 86, 075154 (2012).

11. Gardner, J. S. et al. Cooperative paramagnetism in the geometrically frustrated pyrochlore antiferromagnet tb2ti2o7. Phys. Rev. Lett. 82, 1012-1015 (1999).

12. Thompson, J. D. et al. Rods of neutron scattering intensity in $\mathrm{yb}_{2} \mathrm{ti}_{2} \mathrm{O}_{7}$ : compelling evidence for significant anisotropic exchange in a magnetic pyrochlore oxide. Phys. Rev. Lett. 106, 187202 (2011).

13. Ross, K. A. et al. Dimensional evolution of spin correlations in the magnetic pyrochlore $\mathrm{yb}_{2} \mathrm{ti}_{2} \mathrm{O}_{7}$. Phys. Rev. B 84, 174442 (2011).

14. Kimura, K. et al. Quantum fluctuations in spin-ice-like $\mathrm{pr}_{2} \mathrm{Zr}_{2} \mathrm{O}_{7}$. Nat. Commun. 4, 1934 (2013).

15. Migdal, A. B. Interaction between electrons and lattice vibrations in a normal metal. Sov. Phys. JETP 7, 996 (1958).

16. Hewson, A. C. The Kondo Problem to Heavy Fermions (Cambridge University Press, Cambridge, UK, 1993).

17. Ohtomo, A. \& Hwang, H. Y. A high-mobility electron gas at the laalo3/srtio3 heterointerface. Nature 427, 423-426 (2004).

18. Reyren, N. et al. Superconducting interfaces between insulating oxides. Science 317, 1196-1199 (2007).

19. Mannhart, J. \& Schlom, D. G. Oxide interfaces—an opportunity for electronics. Science 327, 1607-1611 (2010).

20. Coleman, P. Introduction to Many Body Physics (Cambridge University Press, Cambridge, UK, 2015).

21. Doniach, S. The kondo lattice and weak antiferromagnetism. Physica $B+C$ 91, 231-234 (1977).

22. Coleman, P. \& Andrei, N. Kondo-stabilised spin liquids and heavy fermion superconductivity. J. Phys. Condens. Matter 1, 4057 (1989).

23. Senthil, T., Sachdev, S. \& Vojta, M. Fractionalized fermi liquids. Phys. Rev. Lett. 90 , 216403 (2003).

24. Lee, D. H. \& Zimanyi, G. T. New superconducting state of the anderson-lattice model. Phys. Rev. B 40, 9404-9407 (1989).

25. Senthil, T., Vojta, M. \& Sachdev, S. Weak magnetism and non-fermi liquids near heavy-fermion critical points. Phys. Rev. B 69, 035111 (2004).

26. Shankar, R. Renormalization-group approach to interacting fermions. Rev. Mod. Phys. 66, 129-192 (1994).

27. Henley, C. L. Power-law spin correlations in pyrochlore antiferromagnets. Phys. Rev. B 71, 014424 (2005).

28. Isakov, S. V., Gregor, K., Moessner, R. \& Sondhi, S. L. Dipolar spin correlations in classical pyrochlore magnets. Phys. Rev. Lett. 93, 167204 (2004).

29. Conlon, P. H. \& Chalker, J. T. Spin dynamics in pyrochlore heisenberg antiferromagnets. Phys. Rev. Lett. 102, 237206 (2009).

30. Ryzhkin, M. I., Ryzhkin, I. A. \& Bramwell, S. T. Dynamic susceptibility and dynamic correlations in spin ice. Europhys. Lett. 104, 37005 (2013).

31. Snyder, J. et al. Low-temperature spin freezing in the $d y_{2} \mathrm{ti}_{2} \mathrm{O}_{7}$ spin ice. Phys. Rev. $B$ 69, 064414 (2004).

32. Lee, S.-S., Lee, P. A. \& Senthil, T. Amperean pairing instability in the $\mathrm{u}(1)$ spin liquid state with fermi surface and application to $k$-(BEDT-TTF $)_{2} \mathrm{Cu}_{2}(\mathrm{CN})_{3}$. Phys. Rev. Lett. 98, 067006 (2007).

33. Metlitski, M. A., Mross, D. F., Sachdev, S. \& Senthil, T. Cooper pairing in non-fermi liquids. Phys. Rev. B 91, 115111 (2015).

34. Sigrist, M. \& Ueda, K. Phenomenological theory of unconventional superconductivity. Rev. Mod. Phys. 63, 239-311 (1991).

35. Leggett, A. J. Diatomic Molecules and Cooper Pairs 13-27 (Springer, Berlin, Heidelberg, 2015).

36. Sato, M. Topological properties of spin-triplet superconductors and fermi surface topology in the normal state. Phys. Rev. B 79, 214526 (2009).

37. Qi, X.-L., Hughes, T. L. \& Zhang, S.-C. Topological invariants for the fermi surface of a time-reversal-invariant superconductor. Phys. Rev. B 81, 134508 (2010).

38. Maeno, Y. et al. Superconductivity in a layered perovskite without copper. Nature 372, 532-534 (1994).

39. Little, W. A. Possibility of synthesizing an organic superconductor. Phys. Rev. 134, A1416-A1424 (1964).

40. Ginzburg, V. L. The problem of high-temperature superconductivity. ii. Usp. Fiz. Nauk 101, 185 (1970). [Sov. Phys.-Usp. 13, 335 (1970)].

41. Allender, D., Bray, J. \& Bardeen, J. Model for an exciton mechanism of superconductivity. Phys. Rev. B 7, 1020-1029 (1973). 
42. Koerting, V., Yuan, Q., Hirschfeld, P. J., Kopp, T. \& Mannhart, J. Interface-mediated pairing in field effect devices. Phys. Rev. B 71, 104510 (2005).

43. Stephanos, C., Kopp, T., Mannhart, J. \& Hirschfeld, P. J. Interface-induced d-wave pairing. Phys. Rev. B 84, 100510 (2011).

44. Gozar, A. et al. High-temperature interface superconductivity between metallic and insulating copper oxides. Nature 455, 782-785 (2008).

45. Pereiro, J., Petrovic, A., Panagopoulos, C. \& Božović, I. Interface superconductivity: history, development and prospects. Phys. Express 1, 208-241 (2011).

46. Wang, Q.-Y. et al. Interface-induced high-temperature superconductivity in single unit-cell FeSe films on $\mathrm{SrTiO}_{3}$. Chin. Phys. Lett. 29, 037402 (2012).

47. Ahn, C. H., Triscone, J. M. \& Mannhart, J. Electric field effect in correlated oxide systems. Nature 424, 1015-1018 (2003).

48. Ueno, K. et al. Electric-field-induced superconductivity in an insulator. Nat. Mater. 7, 855-858 (2008).

49. Ye, J. T. et al. Superconducting dome in a gate-tuned band insulator. Science $\mathbf{3 3 8}$ 1193-1196 (2012).
Open Access This article is licensed under a Creative Commons Attribution 4.0 International License, which permits use, sharing, adaptation, distribution and reproduction in any medium or format, as long as you give appropriate credit to the original author(s) and the source, provide a link to the Creative Commons license, and indicate if changes were made. The images or other third party material in this article are included in the article's Creative Commons license, unless indicated otherwise in a credit line to the material. If material is not included in the article's Creative Commons license and your intended use is not permitted by statutory regulation or exceeds the permitted use, you will need to obtain permission directly from the copyright holder. To view a copy of this license, visit http://creativecommons. org/licenses/by/4.0/.

(c) The Author(s) 2017 